\title{
Impact of disruption between options of plutonium multi-recycling in PWRs and in SFRs
}

\author{
Jiali Liang ${ }^{1}$, Marc Ernoult ${ }^{1, *} \mathbb{0}$, Xavier Doligez ${ }^{1}$, Sylvain David $^{1}$, and Nicolas Thiollière ${ }^{2}$ \\ ${ }^{1}$ IJCLab, Paris-Saclay University, CNRS/IN2P3, 91405 Orsay, France \\ ${ }^{2}$ Subatech, University of Nantes, IMT Atlantique, CNRS/IN2P3, Nantes 44307, France
}

Received: 15 July 2021 / Received in final form: 17 September 2021 / Accepted: 8 October 2021

\begin{abstract}
During the recent ten years, the estimation of future uranium demands has changed greatly, and SFR competitiveness is called again into question. In this context, a planning of plutonium multi-recycling in PWRs for the near-term decades has been announced in France, which replaces the objective of future SFR deployment. However, the mid-term policy concerning the future reactor system is always uncertain, and the demand of SFR deployment may re-increase significantly. This study looks into this possibility and analyzes the consequences of such back and forth between different plutonium multi-recycling strategies. The newly developed methodology of robustness assessment is applied to the problem, considering the objective disruptions to take into account the deep uncertainties about nuclear future. Two prior trajectories of plutonium multi-recycling, one involving the use of MIX fuel in PWRs and the other considering the SFR deployment, are analyzed first. The disruption of the strategy using MIX is then supposed under the re-consideration of future SFR deployment. To quantify the impacts of using MIX on deployment timing, we investigate the earliest time for which the fleet substitution with SFRs can be completed. To supplement, the prior strategy of SFR deployment is also disrupted under the context of halting the start of new SFR. The plutonium multirecycling in PWRs, regarded as adaptive strategy, aims to minimize the idle plutonium. In these robustness assessments, numerous outputs of interests are analyzed, in order to provide a comprehensive evaluation of consequences of prior strategies, regarding the uncertain disruptions and optimal readjustments.
\end{abstract}

\section{Introduction}

Based on the Act of radioactive materials and wastes management published in 2006 [1] and the specific expertise, French nuclear actors launched the Advanced Sodium Technological Reactor for Industrial Demonstration (ASTRID) project, regarded as the first phase for the future deployment of Sodium-cooled Fast Reactor (SFR) [2]. Following an estimation of global nuclear expansion that would consume rapidly the natural uranium resources $[2,3]$, the planned SFR deployment aimed to build a nuclear fleet independent from the consumption of natural uranium (mainly the fissile ${ }^{235} \mathrm{U}$ ). It might also stabilize or even reduce considerably the stockpiles of spent fuels [4]. In various scenario studies about the massive SFR deployment for the French fleet, a wide range of possible transition strategies has been investigated, concerning the influences on the plutonium quality and global plutonium inventory under different spent fuel management options [5-7], the effects of deployment pace on the natural uranium consumption and wastes packages [8], the

\footnotetext{
*e-mail: marc.ernoult@ijclab.in2p3.fr
}

spent fuel dynamics under possible synergy of European collaborations [9], etc.

During the recent ten years, the political and industrial attitudes towards the SFR technology and the relevant deployment schedule changed a lot. After the Fukushima nuclear accident, the estimation of the nuclear expansion for the coming decades becomes more modest than the optimistic studies in the past, leading to the estimation of rising cost for future new reactor projects [10]. The uranium resources are now considered sufficiently available and the risk of uranium scarcity may remain low for the near-term future [11]. All these factors result in a decreasing demand for SFR technology. In France, the abortion of the ASTRID project was finally declared, after a series of project modifications as well as several project- and organization-related difficulties [12]. It is clarified in the governmental report [13] that the SFR deployment is not considered for the near-term French fleet, even though necessary researches should be carried on to keep the acquisition of relevant knowledge and techniques; instead, the plutonium multi-recycling in Pressurized Water Reactors (PWRs) is considered for the spent fuel management strategies. 
These evolutions reveal the deep uncertainty of nuclear future. One may doubt if prior efforts under a predetermined strategy would bring irreversible regrets in case of objective disruptions. Thus, the influences of uncertain future decisions on the fuel cycle should be taken into account from a physics point of view. The impacts of fuel cycle parameter uncertainties have been investigated through sensitivity analyses which quantify the uncertainties into variation ranges [14-16]. In terms of abrupt changes, a systematic analysis on the disruption of installed capacity has been carried out, developing a dedicated methodology to identifying resistant and resilient strategies in regard to the respect of industrial constraints [17].

Different from these previous studies, this work aims to investigate the deep uncertainty of objectives, and its impact on the deployment of different technologies for the near term before any disruption. The framework of analysis method is presented in detail in Section 2. The notion of robustness is introduced and stands for the capacity of a strategy to adapt to the objective changes. The scenarios of interest are then described in Section 3, inspired from possible transitions of French nuclear fuel cycle: under the deep uncertainty of nuclear future, one may doubt whether the SFR deployment would be reconsidered during the coming decades of plutonium multi-recycling in PWRs in France. We can also imagine that in the situation where future SFR deployment is pursued, the start of new SFRs would be halted in the middle of massive deployment due to economical difficulties of SFRs. The analysis of these two possibilities, though hypothetical, can provide a preliminary investigation on different choices under deep uncertainties for technology development necessary to make them available for plutonium multi-recycling . Correspondingly, the robustness assessment of strategies as well as the physical analyses of the scenarios of interest are performed in Section 4. In both possible cases, a robust strategy is defined as the combination of the prior strategy before disruption and the necessary readjustments achieving the new objective in case of disruption. The work is finally concluded with some outlooks in Section 5.

\section{Framework of study}

A methodology for the robustness assessment of strategies in electro-nuclear scenario studies has been developed in the previous study of [18]. In this methodology, the disruption, which stands for an abrupt change without clear description of probability, is used as the approach to integrate the uncertainties of objective for the nuclear future. The general framework of this methodology is applied to the problem in this work.

The scenario study in [18] investigates the global sensitivity of parameters in a large exploratory space based on statistical approach, only focusing on one single output metric for each objective. In this work, the optimization approach is employed for adaptation scenarios in order to focus on a small number of adaptive strategies. The analyses on several outputs and the related evolution can then be carried out for a comprehensive assessment.

\subsection{Possible strategies before any disruption}

This study is inspired from some possible French national strategies, based on the French nuclear fuel cycle. Learning from previous studies, there are mainly two technological orientations for the French nuclear future: the plutonium multi-recycling in PWRs and in SFRs. These two orientations are to some degree contradictory in the current situation, because the plutonium multi-recycling in PWRs aims to stabilize the plutonium in the cycle, whereas a massive deployment of SFRs requires a much larger accumulation of plutonium than the current inventory. Based on this principle, two strategies and the respective trajectories are first analyzed.

The first trajectory is denoted as TRJ MIX, in which the MIX fuel design is used to multi-recycle the plutonium in PWRs, coherent with the reference strategy illustrated in [13]. MIX fuel is an advanced fuel design that adds enriched-uranium UOX homogeneously to plutonium oxide coming from spent MOX fuel, in order to compensate the deterioration of plutonium quality due to the multi-recycling in PWRs [19]. In the fresh MIX fuel, the plutonium content is preset (in current estimation close to $8 \%$ due to safety constraints), and the uranium enrichment is adjusted according to the plutonium quality and the target burn-up. MIX concept shall be differentiated from MOXEUS concept, very similar except that the plutonium content is not preset but capped. Other fuel assembly designs can be also considered for the multirecycling in PWRs in an industrial scale, such as CORAIL assembly $[19,20]$. Here the MIX is used to represent any pathway of plutonium multi-recycling in PWRs because the goal is not to perform the systematic comparison between diverse fuel designs. The objective of deploying this technology is to stabilize the plutonium inventory in the cycle and to lighten the burden on the spent fuel storage without deploying the SFR.

The second one, denoted as TRJ SFR, suggests an accelerated SFR deployment for $100 \%$ of the French fleet, inspired from [6]. The SFR deployment used to be a reference strategy in France in the past [2]. The French design of SFR uses plutonium for its fresh MOX fuel fabrication. For the sake of simplicity and clarity, the notion of MOX in this paper denotes only the MOX of PWR.

Figures $1 \mathrm{a}$ and $1 \mathrm{~b}$ show respectively the configuration of fuel cycle of these two trajectories, TRJ MIX and TRJ SFR. The arrows explain the orders of spent fuels reprocessing to recycle the plutonium for the advanced fuel fabrication (MIX or SFR fresh fuel). In both cases, the reprocessing of spent PWR MOX fuels are always in priority because its significant accumulation in the current fuel cycle is the first issue to be tackled. For the fresh MIX fuel, the plutonium from spent MIX fuels come to the second while the spent UOX are also reprocessed if more plutonium is demanded. In TRJ SFR, the reprocessing of spent SFR fuels starts after reprocessing all spent fuels discharged from PWRs.

These two trajectories correspond to different objectives and future states of the French nuclear fuel cycle in the current context. They are prior to any future disruption, and thus called prior trajectories in this study. For the 


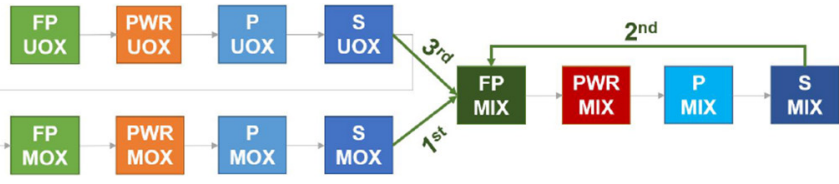

(a) Fuel cycle in TRJ MIX

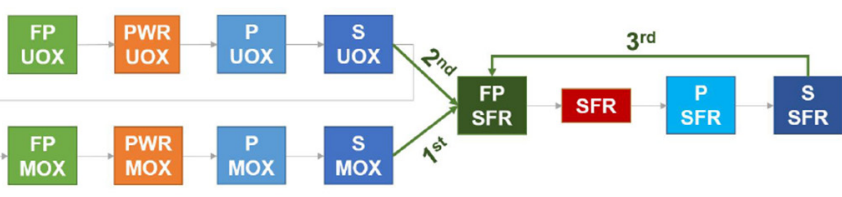

(b) Fuel cycle in TRJ SFR

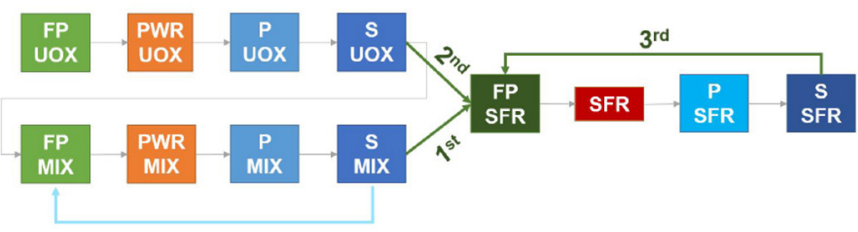

(c) Fuel cycle in SCN MIX2SFR

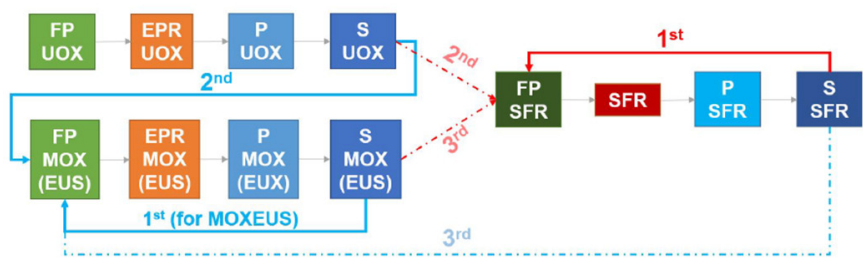

(d) Fuel cycle in SCN SFR2MOXEUS

Fig. 1. Fuel cycles of interest in TRJ MIX, TRJ SFR, SCN MIX2SFR and SCN SFR2MOXEUS. The acronyms in the figures: FP means fabrication plant, $\mathbf{P}$ means cooling pool, $\mathbf{S}$ means interim stock for spent fuels after necessary cooling. Arrows highlighted with orders stand for the priority of spent fuel reprocessing to recycle the plutonium for the advanced fuel fabrication.

following analyses, it is also important to consider a baseline reference for comparison regarding these two prior trajectories. Here the continuation of current technology, a fleet composed of $90 \%$ of PWR UOX and $10 \%$ of PWR MOX for the plutonium mono-recycling, is also simulated. This trajectory is referred as TRJ FrMono.

\subsection{Uncertain disruptions and possible adaptations}

The objectives may evolve following the changes of policies and nuclear perspectives, while these changes are deeply uncertain. To integrate these uncertainties, each aforementioned future state is supposed to be disrupted.

The disruption of TRJ MIX can be triggered by the re-estimation of uranium scarcity, which considers again the risk of future uranium shortages. The post-disruption adaptation scenario, denoted as SCN MIX2SFR, is supposed to reconsider the SFR deployment. The fuel cycle is actually very similar to that in TRJ SFR, as presented in
Figure 1c. The new objective after disruption is to investigate whether the SFR deployment for 100\% fleet can finish as early as TRJ SFR in which the plutonium is never multi-recycled in PWRs. If it is, the pre-disruption strategy using MIX fuels in TRJ MIX can then be adapted to the new objective after disruption, and thus its combination with appropriate adaptations is considered adaptively robust.

The disruption of TRJ SFR may be caused by the unexpected engineering issues or the changes of economic competitiveness in SFR technology. The post-disruption scenario, denoted as SCN SFR2MOXEUS, is supposed to halt the start of new SFRs in the phase of massive SFR deployment. Without new SFRs put into service, it is possible to accumulate the plutonium in interim stocks that is out of use. The interest of post-disruption adaptation is to maximize the use of plutonium, or equivalently, minimize the idle plutonium remained in interim stocks. The plutonium multi-recycling in PWRs is considered for adaptive strategies after disruption. Nevertheless, the SFRs in operation will not be shut down immediately for economic reasons, and they will continue their service until their lifetime limit.

MOXEUS fuel [21] is used instead of MIX for the plutonium multi-recycling in PWRs because it allows more flexibility for fuel fabrication. In TRJ SFR plutonium to be loaded in multi-recycling PWRs may come from spent SFR fuels whose quality is high enough not to need any mix with enriched uranium. MIX fuels do not allow such possibility.

The fuel cycle is presented in Figure 1d. After the disruption, the fleet of all SFRs is supposed to be a single system of SFR-fleet, and the fleet of all EPRs loaded with UOX or MOXEUS is supposed to be another system of EPR-fleet after the disruption. The dotted lines stand for the situation that, for instance, the plutonium in spent SFR fuels is not sufficient for the fabrication of fresh SFR fuels; then the reprocessing of spent UOX or spent MOXEUS fuels are considered for the fresh SFR fuel fabrication. It is the same case for the fresh MOXEUS fabrication. Even though these two sub-fleets using different technologies are supposed to be separate, the use of plutonium is still flexible under these connections represented by the dotted lines.

In SCN SFR2MOXEUS, the maximal idle plutonium in interim stocks after adaptations (under optimization) will be compared with the stable inventory in TRJ MIX in which the plutonium is not accumulated for SFR deployment. If this maximal level after adaptations is lower than that in TRJ MIX, the pre-disruption strategy of SFR deployment in TRJ SFR can then be adapted to the requirement of idle plutonium minimization, and thus its combination with relevant adaptive strategy is considered adaptively robust.

It is worth noting that the outcome in one prior trajectory is used as a threshold to assess the strategy robustness for another one with post-disruption readjustment planning. In fact, the robustness assessed here indicates whether the regrets from the implemented predisruption strategy can be absorbed by post-disruption 
adaptations, relative to the other pre-disruption choice that has never been implemented.

\subsection{Nelder-Mead optimization: identifying optimal adaptive strategies after disruption}

Optimization approach is considered to identify optimal adaptive strategies regarding the given output in two adaptation scenarios. In this work, the NelderMead method, a simplex-based single-output optimization approach is used [22].

This method allows to find the set of inputs, written as an $n$-dimensional vector $\boldsymbol{X}$, which lead to a minimum value of the one-dimensional output $y=f(\boldsymbol{X})$. The numerical algorithm depends on four coefficients described in [22]: $\alpha$ which drives reflections of the simplex during the optimization, $\gamma$ which drives its expansion, $\beta$ drives its contractions and $\delta$ drives its shrinkage. They are subject to:

$$
\alpha>0, \gamma>1, \gamma>\alpha, 0<\beta<1,0<\delta<1 .
$$

In this study, standard values as suggested in [23] are used:

$$
\alpha=1, \gamma=2, \beta=\frac{1}{2}, \delta=\frac{1}{2} .
$$

Note that this optimization algorithm does not incorporate directly the additional constraints. In this study, the strategies that lead to plutonium shortages for fresh fuel fabrication before the end of scenario are considered invalid. To take this into account, penalties on the output as suggested in [22] are used (addition of a given value on the output). Moreover, the algorithm does not guarantee the exploration of all optima in the space. This should be kept in mind so that the results of optimal adaptations should not be over-interpreted.

\subsection{Simulator CLASS and macro-reactor assumption}

The code CLASS is used for the dynamic simulation of nuclear fuel cycle in this study [24]. This simulator applies an artificial neural network approach for the fuel loading models and irradiation models. Currently no limit is imposed on the fabrication and reprocessing capacities. The mass flows of reprocessing towards fabrication plants are supposed to respond to the demand of fuel fabrication and to be transported instantly.

The macro-reactor assumption is applied in this study. It means that all same fuel type of the same reactor type are represented by a single reactor, normalized by the total power level of this fuel/reactor type. For instance, the PWRs using UOX fuels in the fleet are simulated by one PWR UOX, while the parts using MOX are simulated by another one PWR MOX. PWR UOX and PWR MOX models are completely independent. Consequently, all the loadings and discharges of reactors using the same type of fuel occur at the same time in the simulation. This simplification may introduce some biases mainly concerning the synchronization [25]. Nevertheless, we consider that the impacts on the inventory analyses in this study are relatively small compared to the gain on the simulation and the analyses.

\section{Scenario description}

The scenarios of interest start in year 2015 and end in 2160, a time far in the future that allows the long-term evaluation of post-disruption adaptations. Based on the historical operation of French reactors and the future transitions of interest, the individual reactors are normalized into macro-reactors in the following simulations: PWR UOX, PWR MOX, PWR MIX, PWR MOXEUS and SFR. In 2015, the total thermal power of the simulated fleet is 188.1 GWth, contributed by the fleet of PWR UOX and PWR MOX. A loading factor of $72.8 \%$ averaged from the overall historical trajectory is used to transform the nominal power to the effective power.

\subsection{Common transition until the hinge time year 2040}

The assumptions of prior trajectories of interest are schematized in Figure 2. As mentioned in Section 2, TRJ FrMono, the trajectory considering the current technology, i.e. the fleet of $90 \%$ PWR UOX and 10\% PWR MOX, is simulated. The two trajectories using advanced designs, TRJ MIX and TRJ SFR, start diverging from the hinge time, year 2040. These two trajectories stand for the diverse technological orientations for the nuclear future.

Before 2040, the fleet is only composed of PWR UOX and PWR MOX. The total installed capacity is supposed to decrease linearly to $75 \%$ of its initial level. This reduction corresponds to the decommissioning of some old-generation PWRs, compatible with the planning of decreasing nuclear share in the total electricity generation in France [13]. In this study, the transition concerning the total installed capacity is considered from year 2027 to year 2035. After that, the installed capacity of the fleet is supposed constant until the end of scenario regardless of the fuel design or technology deployed afterwards.

After 2040, EPRs are supposed to replace the old PWRs which reach their life limit, so that the installed capacity of fleet can be maintained constant. Even though they employ the same physics models in CLASS, PWRs and EPRs are distinguished in the subsequent simulations. In fact, CLASS simulates the thermal power instead of electric power, and the thermal efficiency of different reactor technologies should be considered here to respect the constant fleet installed capacity. For the current PWRs, the efficiency is supposed to be $33 \%$, while it is $35 \%$ for EPRs and $40 \%$ for SFRs. Therefore, considering the replacements of old PWRs after 2040, the thermal power of the simulated fleet changes slightly.

The replacement of old PWRs with new EPRs is supposed to finish in 2060. After that, the burn-up of UOX is supposed to be $55 \mathrm{GWd} / \mathrm{t}$, higher than the current value of $45 \mathrm{GWd} / \mathrm{t}$. 


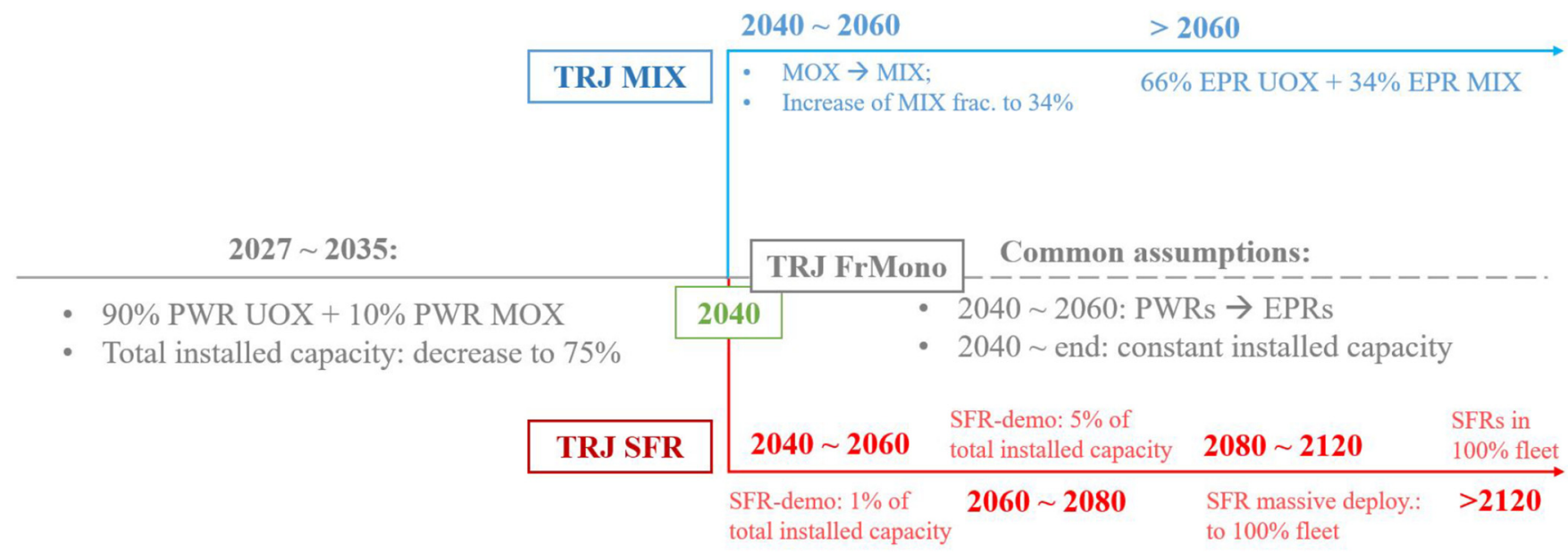

Fig. 2. Description of prior trajectories of interest: TRJ FrMono, TRJ MIX and TRJ SFR.

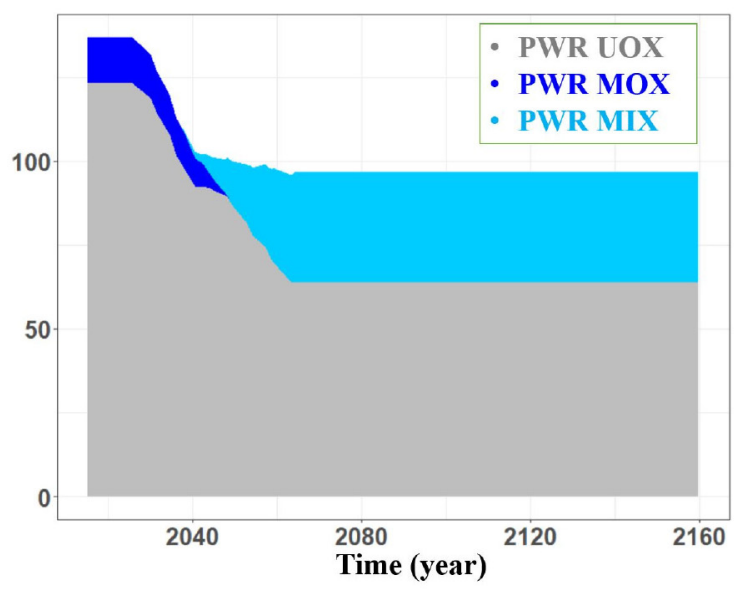

(a) Thermal power evolution in TRJ MIX

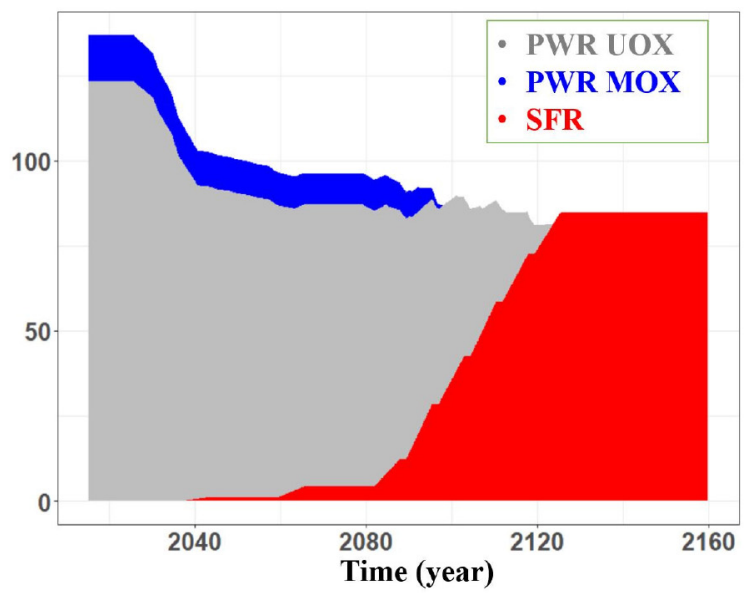

(b) Thermal power evolution in TRJ SFR

Fig. 3. Thermal power evolution in two prior trajectories TRJ MIX and TRJ SFR (in GWth).

\subsection{Prior trajectories of interest}

TRJ FrMono sets a baseline of comparison for the analysis, in which the plutonium is mono-recycled. As indicated in Figure 2, the fleet is approximately constant after the transition of the installed capacity reduction, except for slight adjustments of burn-ups and thermal efficiency.

After year 2040, the trajectories of TRJ FrMono, TRJ MIX and TRJ SFR diverge. For TRJ MIX, the model of MOXEUS in CLASS [26] is used to simulate the MIX fuels. The plutonium content in fresh MIX fuels is set to $9.54 \%$, which is the medium level suggested in [20]. The burn-up of MIX is $55 \mathrm{GWd} / \mathrm{t}$. As indicated in Figure 2, MOX fuels in PWRs are replaced by MIX fuels starting in 2040. At each MOX discharge a new MIX fresh fuel is introduced. The MIX fraction then continues to increase, reaching $34 \%$ in 2060. The thermal power evolution of the fleet is presented in Figure 3a, smoothed by 6-year average. A slight decrease of thermal power between 2040 and 2060 can be observed, which can be explained by the higher efficiency of EPRs than that of PWRs as supposed.
For TRJ SFR, the fleet transition is principally represented by four phases as presented in Figure 2. The first SFR is supposed to start its operation from 2040, accounting for $1 \%$ of total installed capacity. It is regarded as the industrial demonstration for SFR technology. In 2060, new SFRs start their service, and the SFR fraction in the total installed capacity reaches $5 \%$. The massive deployment of SFRs starts from 2080, and the fleet is completely substituted with SFRs by $2120.10 \%$ as the PWR MOX fraction in the part of PWR-fleet is kept till 2085, and decrease to zero by 2095. The thermal power evolution of the fleet accounted by different reactor technologies in TRJ SFR is presented in Figure 3b.

The flexible model in [27] is used to simulate the SFRs, which allows the modeling with different number of blanket layers. Here for the demonstration phase between 2040 and 2060 and the completion phase after 2120, no blanket is added in SFRs; otherwise, two layers of blanket are considered. The burn-up is set to $100 \mathrm{GWd} / \mathrm{t}$ for the fuels in active core when there is no blanket, and the corresponding irradiation time is conserved when adding the 


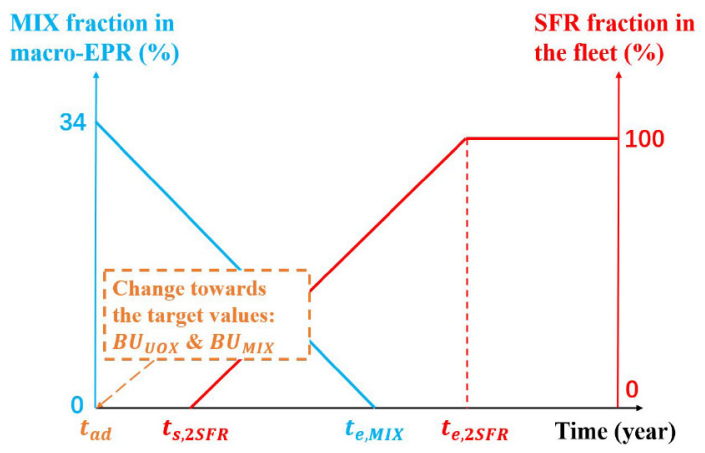

(a) Schema of SCN MIX2SFR

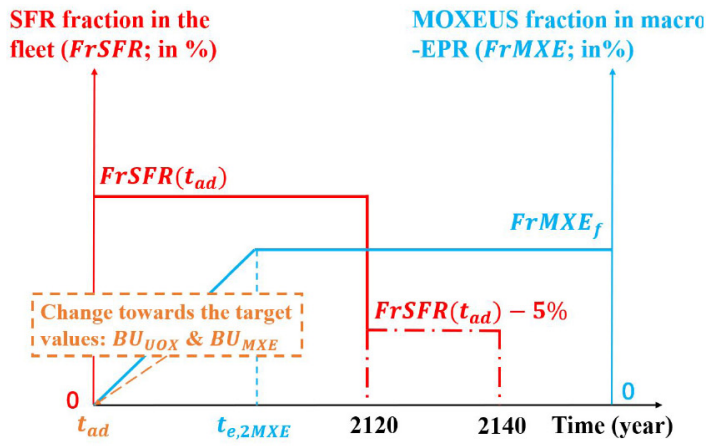

(b) Schema of SCN SFR2MOXEUS

Fig. 4. Description of SCN MIX2SFR and SCN SFR2MOXEUS.

Table 1. Ranges of input variables in the adaptation scenario SCN MIX2SFR.

\begin{tabular}{lllll}
\hline Var. & Min. & Max. & Unit & Explanation \\
\hline$t_{e, \text { MIX }}$ & $t_{a d}+1$ & $\max \left(t_{a d}+1,2095\right)$ & year & $t$ to end the use of MIX. \\
$t_{s, 2 S F R}$ & $\max \left(t_{a d}, 2070\right)$ & 2120 & year & $t$ to start the $1^{\text {st }}$ SFR. \\
$t_{e, 2 S F R}$ & 2120 & 2150 & year & $t$ to reach 100\% SFR. \\
$B U_{U O X}$ & 30 & 60 & $\mathrm{GWd} / \mathrm{t}$ & Burn-up of UOX after $t_{a d}$. \\
$B U_{M I X}$ & 30 & 60 & $\mathrm{GWd} / \mathrm{t}$ & Burn-up of MIX after $t_{a d}$. \\
\hline
\end{tabular}

blankets. This increase mass of uranium in the core due to the blanket without any change in total power or irradiation time, leads to a small transfer of burn-up, blankets assuring part of the power of the irradiation specially at the end of the irradiation. This transfer lead to a slight lower burn-up in the active core and a small burn-up, up to $15 \mathrm{GWd} / \mathrm{t}$, of the blankets.

It is worth noting that this SFR-deployment schedule, inspired from the study in [6], is globally faster than that in other SFR-related scenario studies. Such rapid pace of deployment may not be completely compatible with technological availability. Here this deployment schedule is chosen to emphasize the difference between this future state and the one in TRJ MIX so that the impacts of relevant disruptions can be highlighted as well. Engineering feasibility is not in priority in this strategy assessment.

\subsection{Adaptive strategy space in SCN MIX2SFR}

To take the deep uncertainty of disruption into account, three adaptation times, denoted as $t_{a d}$, are considered: year 2065, 2085 and 2100. The adaptation scenarios of interest, SCN MIX2SFR and SCN SFR2MOXEUS, are schematized in Figure 4.

The adaptation scenario SCN MIX2SFR starts from the prior trajectory TRJ MIX. As presented in Section 2.2, the interest of the disruption is to reconsider the SFR deployment, and to see if it is possible to finish the deployment by year 2120 as in TRJ SFR in which the plutonium is never multi-recycled in PWRs. The plutonium availability is the primary constraint for SFR deployment. This leads us to think of stopping the use of MIX fuels and scheduling reasonably the deployment of SFRs. In this scenario study, five variables are considered, summarized in Table 1, and their characterization on the fuel cycle are schematized in Figure 4a, explained in the following.

First, the progressive stop of using MIX fuels is characterized by the end time $t_{e, M I X}$, which is considered in the range $\left[t_{a d}, \min \left(t_{a d}+1,2095\right)\right]$. During the period $\left[t_{a d}, t_{e, M I X}\right]$ the MIX fraction decreases linearly, and it is zero after $t_{e, M I X}$. The end time of using MOX in PWRs in TRJ SFR, year 2095, is regarded as the upper bound of $t_{e, M I X}$. If it is adapted later than 2095, one year is considered for this transition.

For the scheduling of SFR deployment, the start time of SFR deployment $t_{s, 2 S F R}$, and the time when SFR fraction in the fleet reaches $100 \%, t_{e, 2 S F R}$, are considered. The first irradiation cycle of SFRs begins at $5 \%$ of installed capacity share at $t_{s, 2 S F R}$. Then the share of SFRs increases linearly to $100 \%$ at $t_{e, 2 S F R}$. In consideration of the time needed for the SFR technology development, $t_{s, 2 S F R}$ cannot be earlier than year 2070. For $t_{e, 2 S F R}$, there is no need to be earlier than year 2120.

Moreover, the burn-up of UOX, $B U_{U O X}$, and that of MIX, $B U_{M I X}$, are considered variable to help search for a more efficient accumulation of plutonium.

These five variables build the space of adaptive transition strategies. It is worth noting that the SFR is not considered variable and the same kind of designs than in TRJ SFR are used. The variable $t_{e, 2 S F R}$ is also the output of study to be minimized, subject to the plutonium availability. The optimization process searches for the minimal $t_{e, 2 S F R}$ combined with other strategy parameters 
Table 2. Ranges of input variables in the adaptation scenario SCN SFR2MOXEUS.

\begin{tabular}{lllll}
\hline Var. & Min. & Max. & Unit & Explanation \\
\hline$F r M X E_{f}$ & 0 & 100 & $\%$ & Final MOXEUS fraction in all EPRs. \\
$t_{e, 2 M X E}$ & $t_{a d}+1$ & 2120 & year & $t$ when MOXEUS frac. in the EPR-fleet reaches FrMXE $E_{f}$ \\
$B U_{U O X}$ & 30 & 60 & $\mathrm{GWd} / \mathrm{t}$ & Burn-up of UOX after $t_{a d}$. \\
$B U_{M X E}$ & 30 & 60 & $\mathrm{GWd} / \mathrm{t}$ & Burn-up of MOXEUS after $t_{a d}$. \\
\hline
\end{tabular}

that does not lead to any plutonium shortage for fresh fuel fabrication within the time horizon of scenario.

\subsection{Adaptive strategy space in SCN SFR2MOXEUS}

The adaptation scenario SCN SFR2MOXEUS starts from the prior TRJ SFR. The principal interest is to maximize the use of plutonium, or as equivalently interpreted in this study, to minimize the idle plutonium in interim stocks by multi-recycling plutonium in EPRs by MOXEUS fuels. To achieve this, the final fraction of MOXEUS fuels in all EPRs, denoted as $F r M X E_{f}$, the time to reach this fraction $t_{e, 2 M X E}$, and the burn-ups of UOX and of MOXEUS can be primary strategic parameters of concern. These four variables are considered for adaptive strategies, summarized with their variation ranges in Table 2. Their roles in SCN SFR2MOXEUS are schematized in Figure 4b.

From adaptation time $t_{a d}$ to $t_{e, 2 M X E}$, the MOXEUS fraction in the EPR-fleet increases linearly to the final value $\operatorname{Fr} M X E_{f}$, and then it is kept constant afterwards. Similar to that in SCN MIX2SFR, burn-ups of UOX and of MOXEUS, denoted respectively as $B U_{U O X}$ and $B U_{M X E}$, are considered variable.

One should note that even though no new SFR is put into service after disruption, those in-operation SFRs built before $t_{a d}$ continue their service and should respect the limit of their lifetime. To simplify the timeline of SFR decommissioning regarding their life limit, we make the assumptions on the SFR power reduction to consider their decommissioning, as presented in Figure 4b:

- if $t_{a d}<2080$, all SFRs keep their operation from $t_{a d}$ to year 2120 , and then all enter their decommissioning phase in 2120 .

- Otherwise, a part of SFR power corresponding to $5 \%$ of total installed capacity is reduced in 2120 , which corresponds to the decommissioning of SFRs deployed before 2080. The remaining is kept constant and drops to zero by 2140 , which corresponds to the decommissioning of those deployed after 2080 .

Each time when the SFR power decreases, the power of EPRs increases in order to keep the constant installed capacity of the fleet. The power of EPR MOXEUS and EPR UOX during the transition of MOXEUS fraction are adjusted subject to this change of total power of EPRfleet.

The robustness of the pre-disruption strategy in TRJ SFR, dedicated to the SFR deployment, is assessed with appropriate adaptive strategies after disruption in SCN SFR2MOXEUS. The strategy robustness in this adaptation scenario refers to the minimization of idle plutonium. Numerically, under a given adaptive strategy, the maximum of idle plutonium during the last 20 years of scenario is considered the output of interest, denoted as $P u_{i d l e, \max }$. Corresponding to the interest of SCN SFR2MOXEUS, $P u_{\text {idle, } \max }$ should be minimized under optimization. This single value of output, instead of the time-dependent evolution, allows the direct comparison between different adaptations.

\subsection{Additional output metrics for the analysis}

To carry out comprehensive strategy assessments, several outputs are investigated in addition to $t_{e, 2 S F R}$ in SCN MIX2SFR and $P u_{\text {idle,max }}$ in SCN SFR2MOXEUS.

The total plutonium inventory including those in the conditioned wastes, denoted as $P u_{t o t}$, should be first investigated. In fact, in case the phase-out strategy is implemented, all plutonium without further use should be conditioned into waste canisters. Moreover, this inventory is principally composed of the plutonium in the cycle excluding the wastes, indicating directly the plutonium availability for the technology concerned.

As a complementary data about the waste, the total minor actinides inventory (MA) (including the one in the wastes and the one still in the cycle), denoted as $M A_{t o t}$, is analyzed as well.

With respect to the strategy performance in SCN SFR2MOXEUS, it is also interesting to analyze the idle plutonium evolution, denoted as $P u_{i d l e}$. This may reveal why a given adaptation is robust or not in SCN SFR2MOXEUS.

Last but not least, the cumulative consumption of natural uranium resources, denoted as $U_{\text {c.c. }}$, is of interest. Indeed, the primary driving force of SFR deployment is being independent from the natural uranium consumption. This output highlights the difference between the plutonium multi-recycling in PWRs and that in SFRs.

\section{Analyses of the scenarios of interest}

\subsection{Analyses of prior trajectories}

The comparison between the outcomes of TRJ MIX and that of TRJ SFR indicates the divergence of these two future states, with regards to the interest of these two strategies for the nuclear future. 


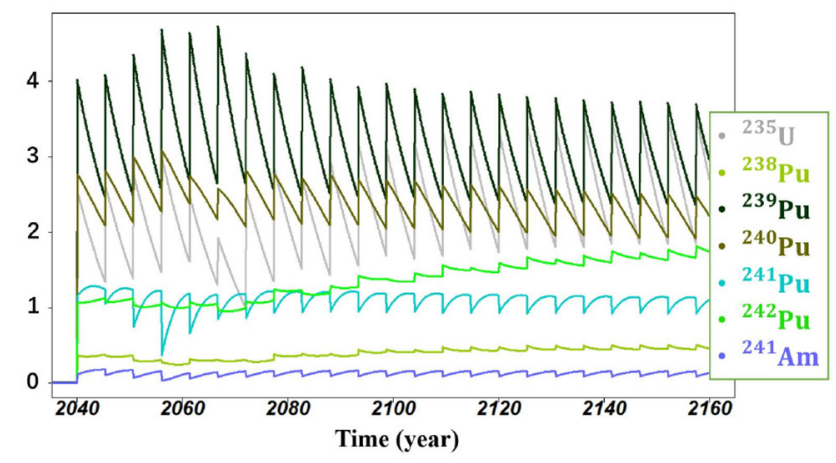

(a) Material content evolution in PWR MIX in TRJ MIX

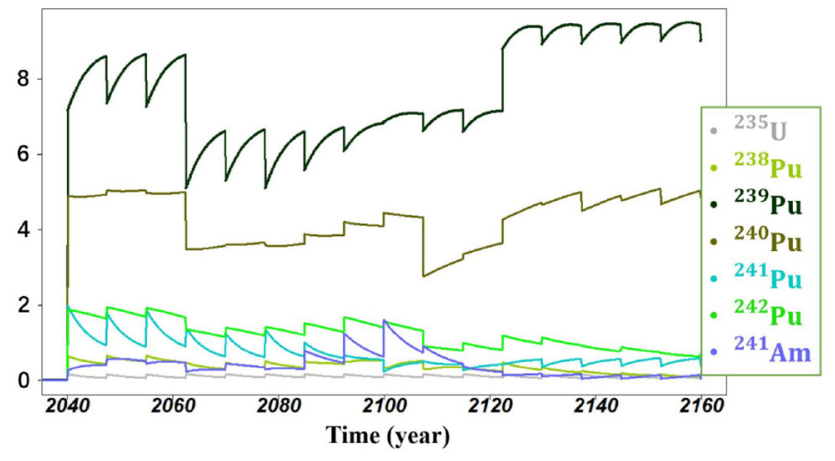

(b) Material content evolution in SFR in TRJ SFR

Fig. 5. Content evolution of plutonium isotopes, ${ }^{235} \mathrm{U}$ and ${ }^{241} A m$ in PWR MIX and in SFR (in \% of the heavy nuclides present in the considered reactor).

First of all, the evolution of some key components in PWR MIX and in SFR gives the fundamental physical explanation for the inventory evolution of interest. The content evolution of plutonium isotopes, ${ }^{235} \mathrm{U}$ and ${ }^{241} \mathrm{Am}$ is shown in Figure 5.

In PWR MIX shown in Figure 5a, the principal plutonium isotope to be incinerated is ${ }^{239} \mathrm{Pu}$, while ${ }^{240} \mathrm{Pu}$ also decreases during the irradiation due to its high content in the fresh fuel and its neutron capture reaction. ${ }^{241} \mathrm{Pu}$ increases under irradiation even though it is fissile. For the ${ }^{241} \mathrm{Pu}$, at the beginning of the irradiation there is small amount since it has decayed before. During irradiation, it is accumulated by Pu240 captures while the disappearance rate is low due to the relatively low quantity. Approximately at the middle of the irradiation cycle, the creation and disappearance rates must be at equilibrium so that the increase in ${ }^{241} \mathrm{Pu}$ mass finishes. The fertile ${ }^{238} \mathrm{Pu}$ and ${ }^{242} \mathrm{Pu}$ accumulate progressively following the multi-recycling in PWRs. This increase of fertile contents in the fresh MIX reduces the plutonium quality and thus increases the ${ }^{235} \mathrm{U}$ enrichment, shown by the gray curve in Figure 5a. An irregular reduction of the ${ }^{235} \mathrm{U}$ enrichment in the 2070s can be observed. That is because the stocks of spent MOX fuels are nearly emptied and some spent UOX fuels are reprocessed for MIX fabrication, introducing a higher-quality plutonium. After that, the ${ }^{235} \mathrm{U}$ enrichment re-increases, reaching a value between $3 \%$ and $4 \%$, which is close to that of fresh UOX fuels.

For the material contents in SFR presented in Figure 5b, one should note that they are normalized by the total mass including the depleted-uranium blankets. That is why the contents before year 2060 and after 2120 are globally higher than that between 2060 and 2120 .

In SFR, ${ }^{239} \mathrm{Pu}$ increases during the irradiation, regardless of the number of blanket layer. ${ }^{240} \mathrm{Pu}$ tends to reach equilibrium during the irradiation cycles in this century. A significant drop on the ${ }^{240} \mathrm{Pu}$ can be observed in the 2110 s, due to the use of higher-quality plutonium from spent UOX fuels after reprocessing all spent MOX fuels. This low content of ${ }^{240} \mathrm{Pu}$ at the beginning of cycle leads to its increase under irradiation. ${ }^{241} \mathrm{Pu}$ content from spent MOX fuels is relatively high and thus it decreases during the irradiation in this century. When the stock of spent MOX fuels is empty after year 2110 and the plutonium from other spent fuels (spent UOX and spent SFR fuels) are used, the ${ }^{241} \mathrm{Pu}$ content is much lower and tends to be stabilized (or slightly increase). The fertile ${ }^{238} \mathrm{Pu}$ and ${ }^{242} \mathrm{Pu}$ decrease slowly during most of irradiation cycles. For the ${ }^{241} \mathrm{Am}$, a particularly high content in the fresh fuels between 2090 and 2110 is presented. This comes from the particular modeling feature of the fuel loading model in [27] which does not separate ${ }^{241} \mathrm{Am}$ from the plutonium for the fuel fabrication. In fact, Last in First out is considered for the spent fuel reprocessing. During these fuel cycles between 2090 and 2110, the oldest spent MOX fuels are reprocessed, in which most of ${ }^{241} \mathrm{Pu}$ decay to ${ }^{241} \mathrm{Am}$. After 2120, spent fuels stay very short time in interim stocks, and thus ${ }^{241} \mathrm{Am}$ content introduced into the fresh fuel fabrication is very low.

This preliminary analysis facilitates the study of global inventories in different prior trajectories. TRJ FrMono provides a reference of comparison in this analysis of TRJ MIX and TRJ SFR. The evolution of four outputs in these three trajectories are presented in Figure 6: $P u_{\text {tot }}$, $M A_{\text {tot }}, P u_{\text {idle }}$ and $U_{\text {c.c. }}$.

As shown in Figure 6a, the plutonium multi-recycling by MIX in $34 \%$ of EPRs is able to stabilize the plutonium in the total cycle. The stabilization is reached from 2060 at around 550 tons. It is consistent with the reference studies such as [20] (with the adjustments on the installed capacity of the fleet). The plutonium inventory in the $100 \%$ fleet of SFRs always increases over time, but has a slower accumulation rate than the fleet in TRJ FrMono. This result highlights the contradiction between two different orientations: the deployment of SFRs in the whole fleet in TRJ SFR demands a large quantity of plutonium in the cycle, while the use of MIX in TRJ MIX limits the accumulation of plutonium.

Figure $6 \mathrm{~b}$ shows that $M A_{t o t}$ is accumulated faster by the using of MIX than that in TRJ FrMono. ${ }^{242} \mathrm{Pu}$ in MIX increases over the multi-recyling. As a result, more and more MA are created. It is worth noting that the $M A_{t o t}$ in the next century is comparable to $P u_{t o t}$ in TRJ MIX, which is much different from the situation 


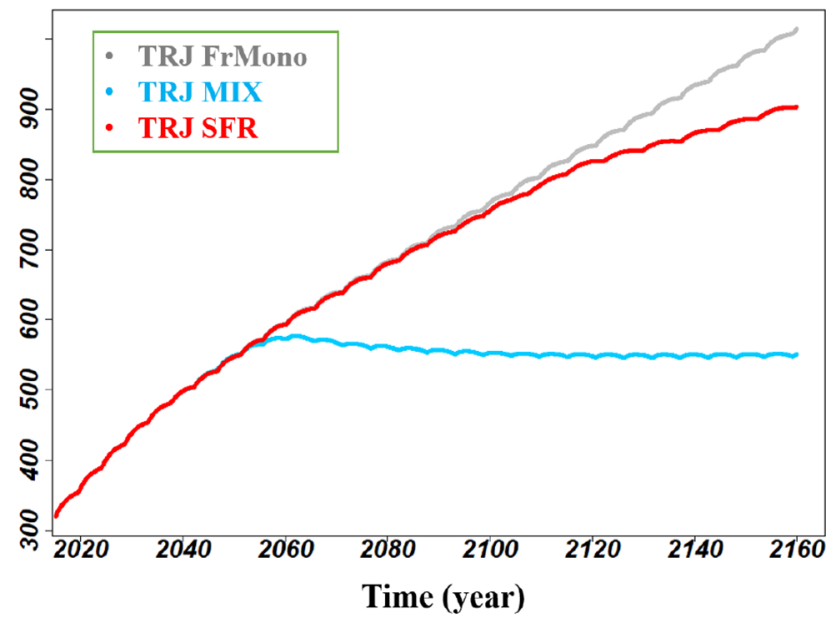

(a) Evolution of $P u_{t o t}$

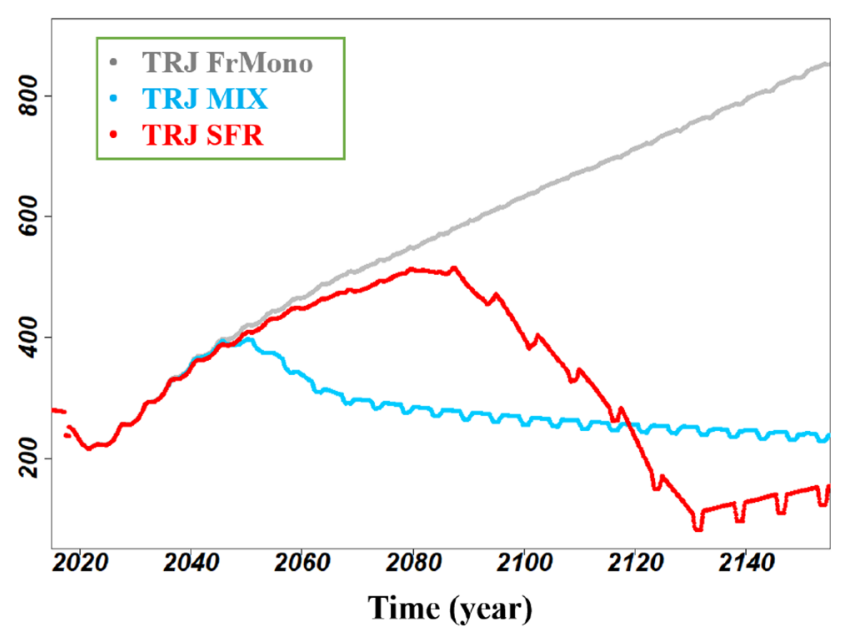

(c) Evolution of $P u_{i d l e}$

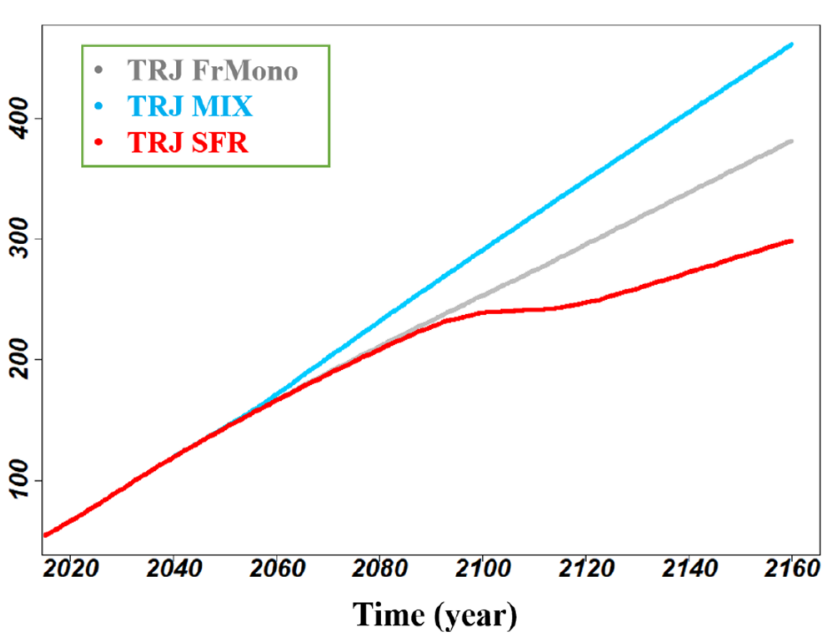

(b) Evolution of $M A_{t o t}$

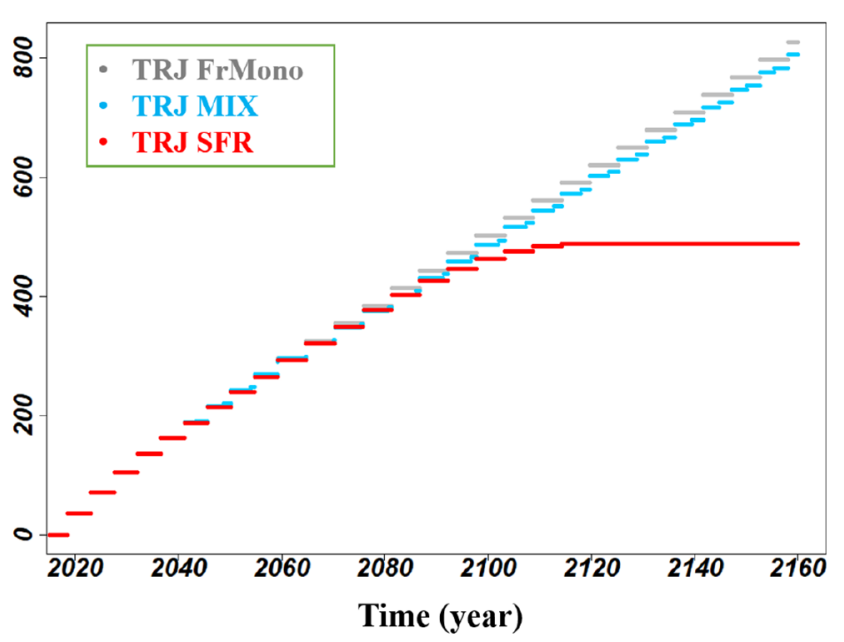

(d) Evolution of $U_{\text {c.c. }}$

Fig. 6. Evolution of $P u_{t o t}, M A_{t o t}, P u_{i d l e}$ (in t) and $U_{c . c .}$ (in kt) in the prior trajectories TRJ FrMono, TRJ MIX and TRJ SFR.

in TRJ FrMono and TRJ SFR. This is principally due to the low amount of $P u_{t o t}$ produced in TRJ MIX, far more than due to the higher amount of Minor Actinides produced compared to other trajectories. In contrast, the $M A_{t o t}$ in TRJ SFR seems to reach a temporal stabilization during the massive SFR deployment before 2120 . This stabilization is due to two effect going in the same direction. Firstly in the 2110s, the spent MOX fuel stock is empty and other sources of $\mathrm{Pu}$ with smaller ${ }^{241} \mathrm{Pu}$ content are used leading to a lower production of ${ }^{241} \mathrm{Am}$. Secondly this plutonium is from very old UOX spend fuel where ${ }^{241} \mathrm{Am}$ has built up, leading to loading of fuel with higher ${ }^{241} \mathrm{Am}$ and thus slightly higher incineration rate of ${ }^{241} \mathrm{Am}$. After 2120, $M A_{\text {tot }}$ re-increases but with a lower rate as expected in SFRs.

Figure 6c verifies that after the transition, the use of MIX stabilizes (or slightly decreases) the plutonium in interim stocks at around 250 tons. It was expected because after the transition (year 2060), the dynamics of plutonium in the total cycle is principally accounted by the one under irradiation. The plutonium stabilization in the total cycle is therefore somehow equivalent to its stabilization in interim stocks after the fleet transition. In TRJ SFR, the plutonium is accumulated till year 2080. When the SFRs begins to be massively deployed, the plutonium in the stocks are rapidly consumed. After 2120 when SFRs are deployed for the whole fleet, the idle plutonium is relatively stable with a slight increase over time.

In terms of the consumption of uranium in Figure 6d, TRJ MIX has a very close tendency to TRJ FrMono. The demand of uranium resource for MIX fuel fabrication is actually similar to that for UOX, as shown by the ${ }^{235} \mathrm{U}$ enrichment in Figure 5a. The SFR deployment, on the contrary, is able to free the fleet from this dependency.

\subsection{Robustness assessment in SCN MIX2SFR}

In SCN MIX2SFR, the new transition aims to complete the fleet substitution with SFRs as soon as possible after the disruption of TRJ MIX. Nelder-Mead optimization 
Table 3. Parameters of optimal adaptive strategies minimizing $t_{e, 2 S F R}$ for three $t_{a d}$ in SCN MIX2SFR.

\begin{tabular}{llllll}
\hline$t_{a d}$ & $t_{e, M I X}$ & $t_{s, 2 S F R}$ & $t_{e, 2 S F R}$ & $B U_{U O X}$ & $B U_{M I X}$ \\
\hline 2065 & 2066 & 2077 & 2120 & 47.7 & 59.2 \\
2085 & 2088 & 2087 & 2120 & 31.8 & 42.1 \\
2100 & 2101 & 2118 & 2140 & 48.6 & 54.4 \\
(Unit) & year & year & year & GWd/t & GWd/t \\
\hline
\end{tabular}

is used to minimize the finish time of SFR deployment $t_{e, 2 S F R}$ for three possible adaptation times $t_{a d}$.

Adaptive strategies achieving minimal $t_{e, 2 S F R} \leq 2120$ can be identified for $t_{a d}=2065$ and $t_{a d}=2085$. To avoid unrealistic deployment paces which substitute the whole fleet with SFRs in one or two irradiation cycles, the optimization is re-performed to minimize the start time $t_{s, 2 S F R}$ for these two $t_{a d}$, presetting $t_{e, 2 S F R}=2120$. On the contrary, the minimal $t_{e, 2 S F R}$ identified for $t_{a d}=2100$ is later than year 2120 . The optimal adaptive strategies after this adjustment for $t_{a d}=2065$ and $t_{a d}=2085$ presented in Table 3 and their results are considered for the following analyses.

Above all, $t_{e, 2 S F R}=2120$ for year 2065 and 2085 as $t_{a d}$ means that the fleet substitution for EPRs with SFRs can finish as early as the SFR-deployment strategy in TRJ SFR. In other words, the pre-disruption strategy applying MIX in TRJ MIX with appropriate adaptive plans is adaptively robust if the adaptations are taken before year 2085. But if the adaptation begins from 2100 , it may be too late and the SFR deployment should finish later than 2120. In this case, the difference between the threshold time (year 2120) and the result of optimization (year 2140), can be regarded as the regret of using MIX fuels in PWRs on the SFR deployment schedule under this late disruption and adaptation.

To minimize $t_{e, 2 S F R}$, all these optimal adaptive strategies suggest the immediate stop of using MIX in EPRs $\left(t_{e, 2 S F R}\right.$ very close to $\left.t_{a d}\right)$. It is coherent regarding the accelerated SFR deployment because the immediate stop of MIX allows an efficient accumulation of plutonium for SFR deployment. The reduction of $B U_{U O X}$ may help increase this accumulation rate as well, but it is relatively marginal compared to the decrease of MIX fraction. However, one may also notice that the immediate stop of MIX implies a relatively long period between $t_{e, M I X}$ and $t_{s, 2 S F R}$ in which no advanced fuel is fabricated. The same remark can be made for the reprocessing plant. There can be some incoherence regarding the industrial constraints which may require the continuous operation of the installed facilities for the reprocessing and the advanced fuel fabrication. Reprocessing and fabrication ahead of time, and the storage of fresh fuel may be an coordinated option to tackle this incoherence.

The outcomes of four other outputs of interest from these optimal adaptive strategies are presented in Figure 7. In comparison, the results of TRJ SFR are also presented.
Figure 7a shows the total plutonium which is principally composed of the plutonium in the cycle. Note that the latter indicates the plutonium availability that constrains the installed capacity of SFRs. According to the simulations, the macro-SFR for $100 \%$ fleet (providing approximately 46.5 GWe after year 2035) demands around $350 \mathrm{t}$ of plutonium for one irradiation cycle. In consideration of the necessary time for recycling which is close to the irradiation time, a plutonium inventory of more than $700 \mathrm{t}$ in the cycle is a necessary condition to avoid plutonium shortages for the total fleet of SFRs. Figure 7a indicates the dynamics of fulfilling this condition regarding different $t_{a d}$. All optimal adaptive strategies seem to result in a higher increase rate of plutonium after the respective $t_{a d}$ than that in TRJ SFR. Indeed, this is due to the immediate stop of MIX and $B U_{U O X}<55 \mathrm{GWd} / \mathrm{t}$, whereas there is always 10\% MOX in the EPR-fleet and $B U_{U O X}=55 \mathrm{GWd} / \mathrm{t}$ is applied to PWR UOX in TRJ SFR. This $P u_{t o t}$ evolution reveals that these optimal adaptations can pile up plutonium rapidly over 700 tons before year 2120 with some margins if these readjustments are taken before year 2085. But $t_{a d}=2100$ is too late for this accumulation and therefore the corresponding minimal $t_{e, 2 S F R}$ is far later than year 2120 .

Figure $7 \mathrm{~b}$ indicates that the longer time MIX fuels are used, the higher $M A_{\text {tot }}$ is accumulated. It is coherent with the analysis of prior trajectories. After finishing the fleet substitution, the $M A_{t o t}$ increase rates in all these trajectories seem to be similar. The impact of using MIX before disruption on the MA accumulation can be therefore deduced from these parallel evolutions.

Figure $7 \mathrm{c}$ verifies the plutonium availability in interim stocks. Although the adaptation from year 2085 seems robust, it has a high risk of plutonium shortage after the transition of SFR deployment. This risk occurs as well for the case of $t_{a d}=2100$ when the substitution of fleet finishes after 2140 .

Last but not least, Figure 7d shows that the longer time of using MIX fuels is, the more natural uranium is consumed. For the case of $t_{a d}=2065$ and $t_{a d}=2085$, the consumption rates just after $t_{a d}$ are slightly higher than that in TRJ SFR because the fleet is $100 \%$ EPR UOX during this period. But compared to $t_{a d}=2100$, these two earlier adaptations lead to a much lower cumulative consumption in the end. That is because the SFR deployment starts early in these two trajectories of early $t_{a d}$ and their SFR deployment timelines are close to that in TRJ SFR. On the contrary, the high consumption rate of uranium for $t_{a d}=2100$ is kept until $t_{s, 2 S F R}=2118$. If the availability or the market price of natural uranium meets a large change in the future, this significantly higher need of natural uranium than that in TRJ SFR should be anticipated.

\subsection{Robustness assessment in SCN SFR2MOXEUS}

In SCN SFR2MOXEUS, the new transition strategy aims to maximize the use of plutonium by using MOXEUS fuels in EPRs after the halting of new SFR. Nelder-Mead 


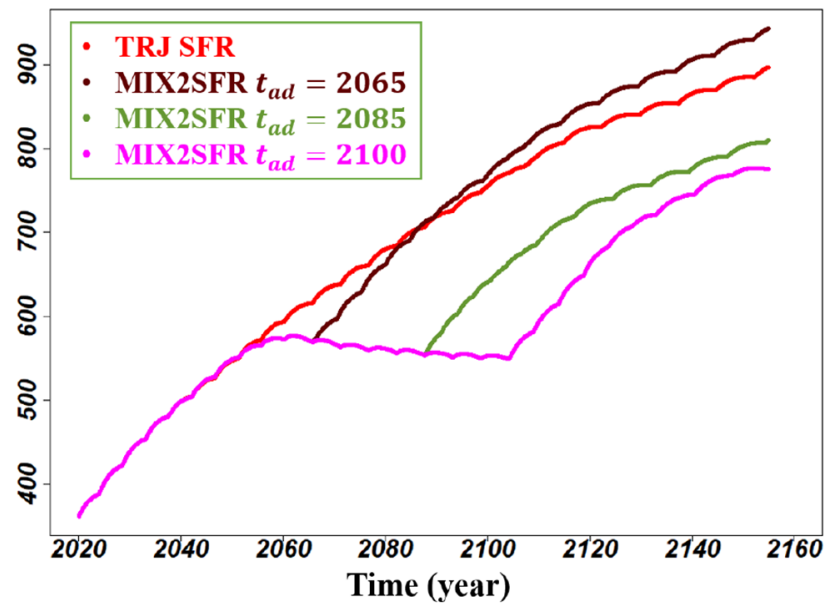

(a) Evolution of $P u_{t o t}$

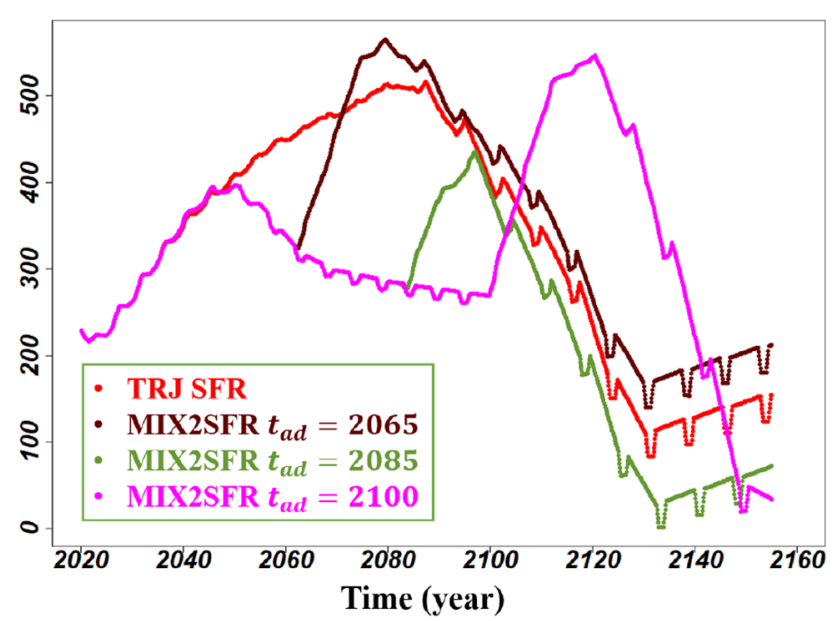

(c) Evolution of $P u_{i d l e}$

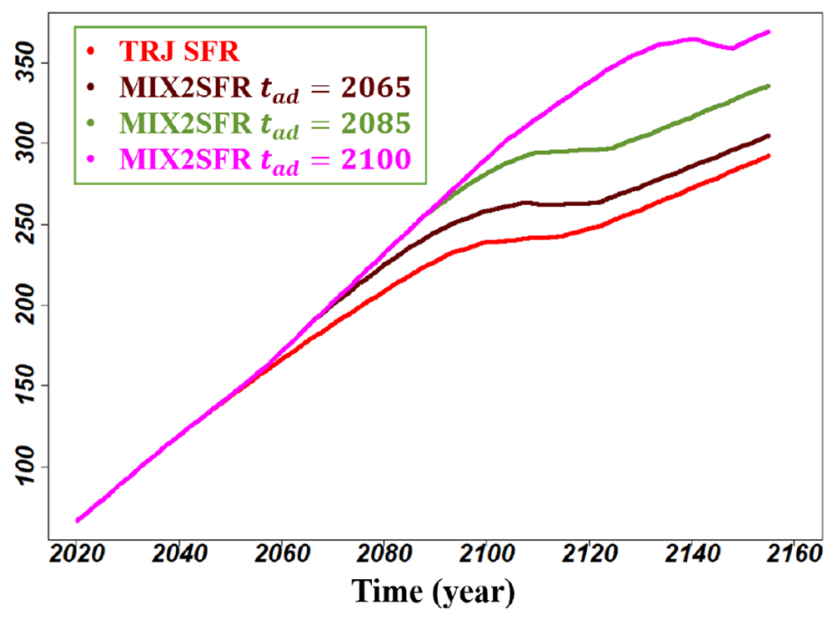

(b) Evolution of $M A_{t o t}$

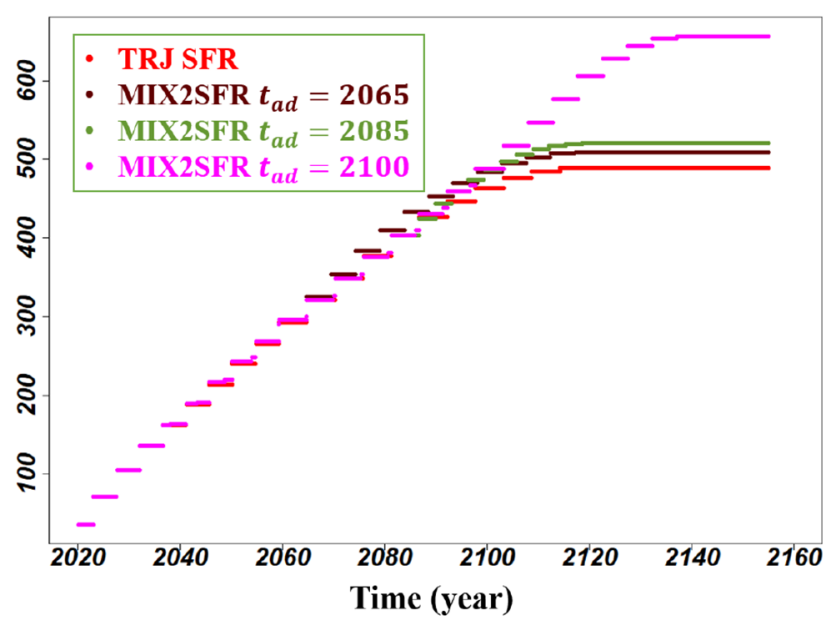

(d) Evolution of $U_{\text {c.c. }}$

Fig. 7. Evolution of $P u_{t o t}, M A_{t o t}, P u_{i d l e}$ (in t) and $U_{c . c .}$ (in kt) from optimal adaptive strategies for three $t_{a d}$ in SCN MIX2SFR, compared with that in TRJ SFR.

Table 4. Parameters of optimal adaptive strategies minimizing $P u_{i d l e, \max }$ for three $t_{a d}$ in $\mathbf{S C N}$ SFR2MOXEUS.

\begin{tabular}{llllll}
\hline$t_{a d}$ & $F r M X E_{f}$ & $t_{e, 2 M I X}$ & $B U_{U O X}$ & $B U_{M X E}$ & $P u_{\text {idle,max }}$ \\
\hline 2065 & 52.8 & 2087 & 40.2 & 37.5 & 17 \\
2085 & 34.3 & 2096 & 40.1 & 36.8 & 174 \\
2100 & 33.4 & 2105 & 38.9 & 40.0 & 332 \\
(Unit) & $\%$ & year & GWd/t & GWd/t & t \\
\hline
\end{tabular}

optimization is applied to minimize the peak of idle plutonium in interim stocks $P u_{\text {idle, } \max }$ during the last 20 years of scenario for three possible $t_{a d}$. The parameters of the optimal adaptive strategies identified are presented in Table 4.

For the robustness assessment, the relatively stable idle plutonium in TRJ MIX in which the plutonium is not accumulated for SFR deployment, at around $250 \mathrm{t}$, is regarded as the threshold level of comparison. Compared to this value, the optimal adaptive strategies of $t_{a d}=2065$ and $t_{a d}=2085$ lead to lower idle plutonium over the last 20 years of scenario. In other words, the strategy of SFR deployment in TRJ SFR with an appropriate adaptive planning is adaptively robust if these appropriate adaptations are taken before year 2085, in regards to the minimization of idle plutonium in interim stocks. But the later adaptation from $t_{a d}=2100$ leads to a higher idle plutonium inventory than the threshold indicated. The excess of this quantity of idle plutonium for $t_{a d}=2100$ can be then regarded as the regret of prior SFR deployment on this new objective under the late disruption and adaptation.

As shown in Table 4, the optimal adaptation of $t_{a d}=$ 2065 suggests a relatively high $\operatorname{Fr} M X E_{f}=52.8 \%$, while the other two suggest lower MOXEUS fractions. This difference of fraction may result intuitively in different material evolution under irradiation. Indeed, even though the MOXEUS fuel design applies similar principles as that of MIX fuel, its variable plutonium content in the fresh 


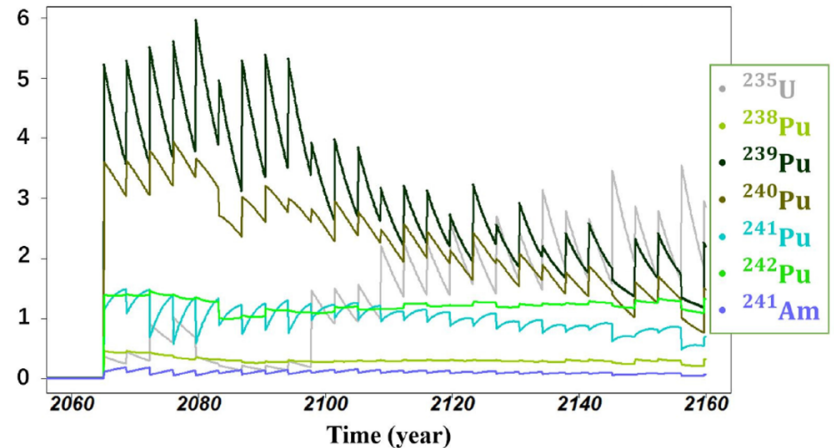

(a) $t_{a d}=2065$

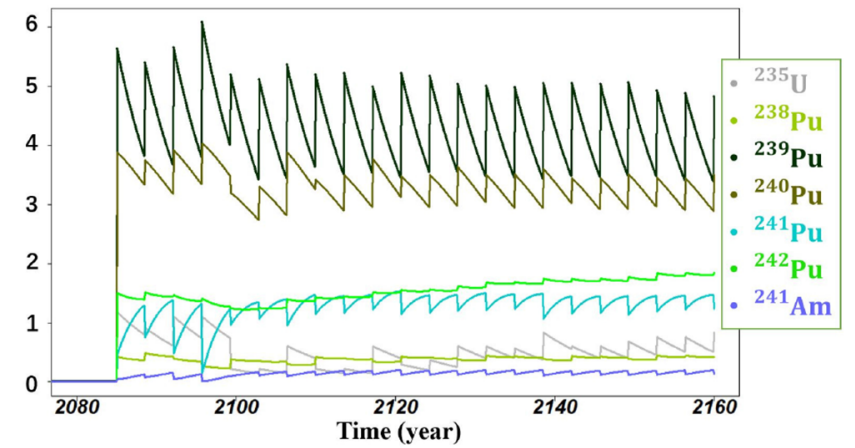

(b) $t_{a d}=2085$

Fig. 8. Content evolution of plutonium isotopes, ${ }^{235} \mathrm{U}$ and ${ }^{241} A m$ in PWR MOXEUS in SCN SFR2MOXEUS for optimal adaptations from year 2065 and 2085 (in \% of the heavy nuclides present in the considered reactor).

fuel can lead to measurable difference on the subsequent evolution from that of MIX fuel. The content evolution of key components in PWR MOXEUS for $t_{a d}=2065$ and $t_{a d}=2085$ are taken for a preliminary analysis, presented in Figure 8.

Some similarities between the material evolution of MOXEUS and that of MIX under irradiation can be observed. The principal incinerated isotope is ${ }^{239} \mathrm{Pu}$, and ${ }^{240} \mathrm{Pu}$ decreases during irradiation. ${ }^{241} \mathrm{Pu}$, mainly produced from the neutron capture of ${ }^{240} \mathrm{Pu}$, increases slowly during irradiation and its mean content is relatively stable during all irradiation cycles.

The measurable difference is that for $t_{a d}=2065$ shown in Figure 8a, the total plutonium content of fresh fuel decreases evidently following the multi-recycling. This can be explained by two factors. On one hand, due to this early adaptation from year 2065, the available plutonium inventory at the beginning of adaptation is lower than those later ones. On the other hand, a higher MOXEUS fraction and a larger share of EPR in year 2065 than that in year 2085 lead to a much faster incineration of plutonium, which results in relatively low available inventory of plutonium for the subsequent fuel fabrication. To achieve the target burn-up, ${ }^{235} \mathrm{U}$ enrichment increases. Between year 2080 and 2100 for $t_{a d}=2065$, the plutonium of higher-quality used for MOXEUS fabrication comes mainly from spent UOX fuels because the stocks of spent MOX are empty and the plutonium inventory from spent MOXEUS is relatively low. After 2100, spent MOXEUS fuels account the most for the plutonium used for fresh MOXEUS fabrication. The lower-quality and the low availability of plutonium for MOXEUS fabrication result together in a sharp increase of ${ }^{235} \mathrm{U}$ enrichment in 2100. All these also explain the extremely low $P u_{i d l e, \max }$ for the optimal adaptive strategies of $t_{a d}=2065$.

In contrast, $t_{a d}=2085$ leads to more plutonium to be used for the start of adaptation, and the lower MOXEUS fraction indicates a lower incineration rate. The evolution of these isotopes of interest in PWR MOXEUS presented in Figure 8bis more similar to that of PWR MIX in Figure 5a, except that the total plutonium content in MOXEUS is averagely higher than in MIX in this case, and thus the ${ }^{235} \mathrm{U}$ enrichment remains much lower (because plutonium content in MOXEUS is variable and adjusted for the burn-up prior to adjusting ${ }^{235} \mathrm{U}$ enrichment).

The evolution of those four output metrics from the optimal adaptive strategies identified are presented in Figure 9. To compare, the outcomes in TRJ MIX are also presented.

Figure 9a verifies that the total plutonium inventory of the optimal adaptation from $t_{a d}=2065$ decreases dramatically. This rapid decrease can be expected, according to the evolution of isotopic contents of plutonium shown in Figure 8a. For the other two $t_{a d}$, even though the MOXEUS fraction is close to the MIX fraction in TRJ MIX, the total plutonium decreases as well, owing to the higher plutonium content in fresh MOXEUS.

In Figure 9b, the identified optimal adaptations from 2065 and from 2085 seem to accumulate more rapidly the $M A_{\text {tot }}$ than that in TRJ MIX. It can be also explained by the higher plutonium content with higher MOXEUS fraction in the EPR-fleet. $M A_{t o t}$ in the trajectory of $t_{a d}=2065$ is even measurably higher than its total plutonium at the end of scenario. The adaptation from year 2100 seems to have a lower increase rate than these two early adaptations. In fact, after year 2100, a large quantity of low-quality plutonium from spent MOX fuels have been used for SFR fuel fabrication. Thus, the plutonium for MOXEUS fabrication after disruption comes mainly from spent UOX fuels, with higher quality and fewer ${ }^{242} \mathrm{Pu}$. This results in a lower plutonium content in fresh MOXEUS. Hence, fewer MA are created during irradiation in this case than other two earlier adaptations.

Figure $9 \mathrm{c}$ reveals the dynamics of $P u_{i d l e}$ under the respective optimal adaptive strategies of different $t_{a d}$, and verifies their peaks $P u_{i d l e, \max }$. For $t_{a d}=2065$, the stocks of idle plutonium are almost empty after 2100, justifying the foregoing low $P u_{\text {idle, } \max }$. For $t_{a d}=2085, P u_{\text {idle }}$ continues decreasing even after finishing the transition by $t_{e, 2 M X E}=2096$, except that a slight increase can be observed after 2140. The re-increase comes from the spent fuel discharge of the last two irradiation cycles of SFRs before being shut down, which are not recycled by SFRs any more. While for $t_{a d}=2100$, these two phases of 


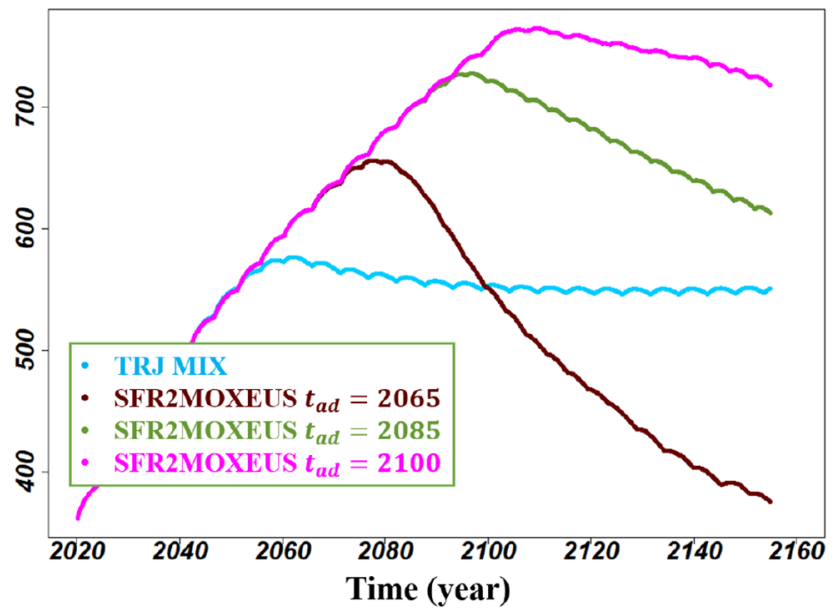

(a) Evolution of $P u_{t o t}$

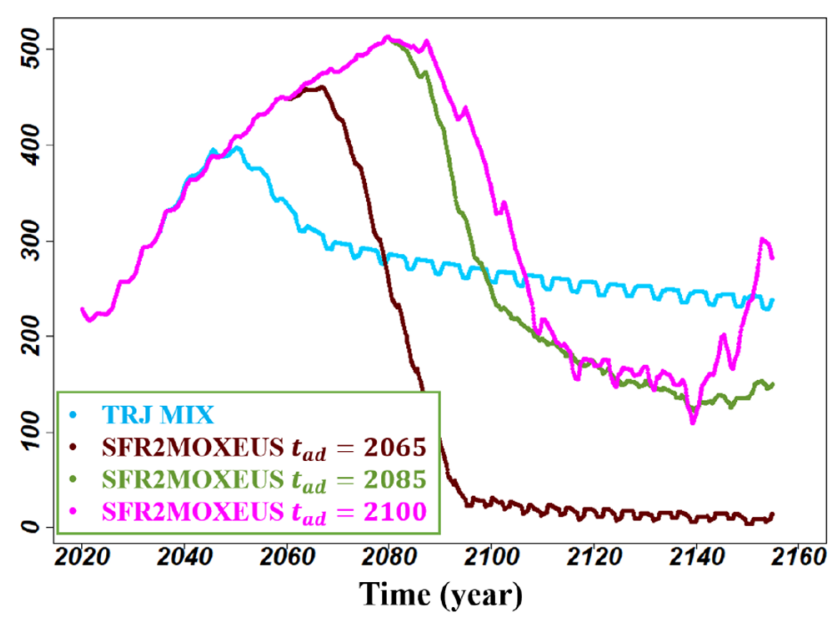

(c) Evolution of $P u_{i d l e}$

Fig. 9. Evolution of $P u_{t o t}, M A_{t o t}, P u_{i d l e}$ (in t) and $U$ SFR2MOXEUS, compared with that in TRJ MIX.

re-increase after year 2140 is much more significant and lead finally to a higher $P u_{i d l e}$ than the threshold of 250 t. Actually, as can be deduced from the power evolution of TRJ SFR in Figure 3b, the SFR share in 2100 is very high, and a large quantity of plutonium is loaded in this macro-SFR. Even though the MOXEUS fabrication demands lots of plutonium, it is still far lower than that of SFRs for the same installed capacity, and at the same time, the corresponding $\operatorname{Fr} M X E_{f}$ is even lower than the SFR fraction in 2100. It reveals that even though the SFR deployment consumes rapidly the idle plutonium in stocks as shown in TRJ SFR, the significant inventory out of use when these reactors enter their decommissioning phase should be taken into account. The difference between this peak, about $332 \mathrm{t}$, and the threshold $250 \mathrm{t}$, can be then regarded as the regret of plutonium accumulation for the SFR deployment. Nevertheless, this peak is still much lower than the global peak before SFR deployment. It can be anticipated in practical industrialization. It is also possible that $P u_{\text {idle }}$ may decrease afterwards, but

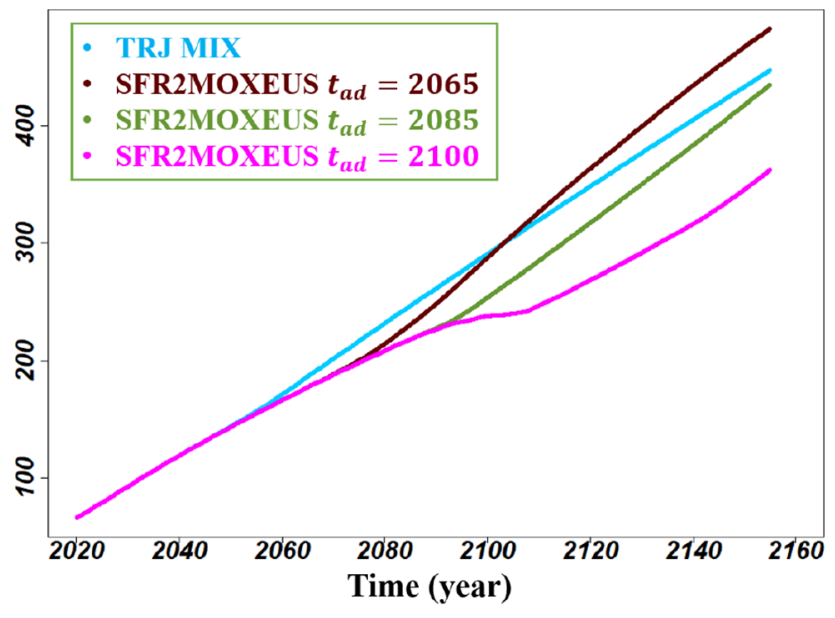

(b) Evolution of $M A_{t o t}$

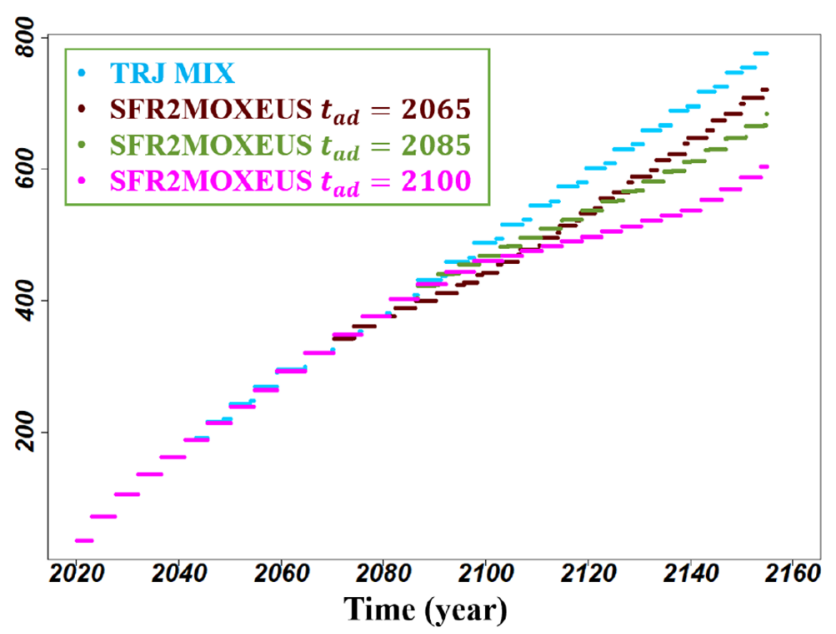

(d) Evolution of $U_{\text {c.c. }}$

(in kt) from optimal adaptive strategies for three $t_{a d}$ in SCN it must need more time and it is out of the time horizon in this study.

Last but not least, Figure 9d shows that the identified optimal adaptations consume fewer natural uranium than the one in TRJ MIX. This can be explained by, on one hand, the operation of SFRs, and on the other hand, the smaller need of ${ }^{235} \mathrm{U}$ enrichment in MOXEUS than that in MIX fuels, due to the priority of varying plutonium content in MOXEUS fabrication.

\section{Conclusion and outlook}

In this work, the methodology of robustness assessment for nuclear fleet transition strategies under the uncertain disruption of objectives, developed in [18], is applied to the assessment of two important strategies inspired from French national strategies: the plutonium multi-recycling in PWRs, and that in SFRs. In this work we defined the robustness of a given strategy as its capacity to be adapted 
to the possible future changes of objectives, reflecting the deep evolution of the nuclear context. The trajectories of applying these two strategies are denoted as TRJ MIX in which the plutonium is multi-recycled by using MIX in $34 \%$ of the future EPR-fleet, and as TRJ SFR in which the SFRs are deployed in $100 \%$ of fleet, respectively. To assess the robustness of these two transition strategies, the disruption in the respective future state is supposed. Three adaptation times are considered: year 2065, 2085 and 2100 .

In this work, the optimization approach allows us to focus on individual strategies of great interest instead of diving into a huge amount of statistical data. Thanks to this, the assessments of strategies of interest can be performed within a wide spectrum of output metrics, potentially linked to diverse future objectives. This provides useful and practical information for decision-making.

The adaptation scenario from TRJ MIX, denoted as SCN MIX2SFR, reconsiders the SFR deployment and aims to minimize the time when the fleet is completely substituted with SFRs. Applying the Nelder-Mead optimization algorithm, optimal adaptive strategies regarding three adaptation times are identified. It shows that the pre-disruption strategy of using MIX with an appropriate adaptive plan can complete the SFR deployment by year 2120, as early as in TRJ SFR, if the appropriate adaptation is taken before 2085; therefore, it is adaptively robust. However, no appropriate adaptation has been found if the disruption occurs in 2100 , so we do not consider it robust. The use of MIX fuels stabilizes actually the plutonium, whereas the operation of SFRs in $100 \%$ of fleet demands a significant quantity of plutonium. The adaptation from 2100 is too late and the piled up plutonium is not sufficient to substitute the whole fleet with SFRs by 2120 .

The adaptation scenario from TRJ SFR, denoted as SCN SFR2MOXEUS, halts the start of new SFRs and aims to minimize the idle plutonium in interim stocks by using MOXEUS fuels in EPRs. Compared to the relatively stable inventory of idle plutonium in TRJ MIX which is regarded as the threshold level, the identified optimal adaptations can lead to lower idle plutonium during the last 20 years of scenario if the adaptation is taken before year 2085. The pre-disruption strategy of SFR deployment with appropriate adaptive plans is therefore considered adaptively robust. However, the optimal adaptation from year 2100 leads to a peak of idle plutonium higher than that threshold. Indeed, the SFR share in the nuclear fleet in 2100 is considerable, and their permanent shut-down releases a significant quantity of spent fuels containing high plutonium content without the future recycling in SFRs. This large quantity of plutonium out of use results then in the regrets on the minimization of idle plutonium.

In addition to the outputs of interest regarding the post-disruption objectives, several output metrics are also considered to assess more comprehensively the strategies of interest. They help indicate the gains and costs of these strategies from different angles, involving the total plutonium and MA inventories in the cycle, the idle plutonium in interim stocks and the cumulative consumption of natural uranium.
For the sake of simplification and calculation cost, the scenario study in this paper is based on some assumptions including notably macro-reactors and no detailed a-priori schedule of reactor deployment. In this case, no life time of individual reactor can be supposed. Note that the lifespan of reactor is an important constraint for industrial application, because it is strongly linked to economic profits and can be an important output metric for policymaking. The cost estimation is not taken into account either. In practise, one may regard economic factors as important contributors to the decision-making process. This academic study only looks into the physical problems concerning the material inventories, but does not considers these economic aspects. Meanwhile, the capacities of spent fuel reprocessing and fresh fuel fabrication, which are also impactful constraints for the deployment of nuclear facilities, are not studied here. Depending on the interests of stakeholders, decision-making actors or analysts, the methodology of robustness assessment applied to this work can still be adapted to the investigation of the output metrics and the constraints aforementioned. To go towards more detailed discussions on national strategies, the next step is to adapt the proposed methodology to relevant industrial issues including the consideration of operational constraints.

Even though the optimization approach is helpful regarding the analyses on a small number of strategies, it cannot indicate the shape of robust adaptive strategy space as the statistical approach does. Especially, there is no clue on the location of the optimized strategy within this space, whether it is surrounded or not by non-valid strategies. If an exploratory study is needed, a systematic comparison between the optimization and the statistical approach in the future can be useful for the method development for scenario studies.

\section{Author contribution statement}

All the authors were involved in the preparation of the manuscript. All the authors have read and approved the final manuscript. Jiali Liang, Marc Ernoult, Xavier Doligez defined the scenarios to be studied. Marc Ernoult, Xavier Doligez and Nicolas Thiollière developed the reactors and fuel loading models used in the simulations. Jiali Liang performed all the calculations.

\section{References}

1. Office parlementaire d'évaluation des choix scientifiques et technologiques (France), The 2006 Programme Act on the Sustainable Management of Radioactive Materials and Wastes (OPECST, 2006)

2. CEA/DEN, La Gestion Durable des Matières Radioactives avec les Réacteurs de 4e Génération (CEA, 2012)

3. E. Mbala Malambu et al., Transition Towards a Sustainable Nuclear Fuel Cycle (NEA-OECD, 2013)

4. CEA/DEN, Inventaire prospectif entre 2016 et 2100 des matières et des déchets radioactifs produits par le 
parc français selon différents scénarios d'évolution (CEA, 2018)

5. L. Tillard, J.B. Clavel, X. Doligez, É. Dumonteil, M. Ernoult, J. Liang, N. Thiollière, Analysis of Transition Scenario from a PWR to a SFR Fleet Simulated with the CLASS code, in International Nuclear Fuel Cycle Conference (GLOBAL 2019), Seattle, Washington, USA (2019)

6. M. Tiphine, C. Coquelet-Pascal, G. Krivtchik, R. Eschbach, C. Chabert, B. Carlier, M. Caron-Charles, G. Senentz, L. Durpel, C. Garzenne, F. Laugier, Simulations of Progressive Potential Scenarios of $\mathrm{Pu}$ Multirecycling in SFR and Associated Phase-out in the French Nuclear Power Fleet, in GLOBAL 2015 - 21st International Conference and Exhibition "Nuclear Fuel Cycle for a Low-Carbon Future", Paris, France (2015)

7. G. Martin, C. Coquelet-Pascal, Symbiotic equilibrium between sodium fast reactors and pressurized water reactors supplied with MOX fuel, Ann. Nucl. Energy 103, 356-362 (2017)

8. D. Freynet, C. Coquelet-Pascal, R. Eschbach, G. Krivtchik, E. Merle-Lucotte, Multiobjective optimization for nuclear fleet evolution scenarios using COSI, EPJ Nuclear Sci. Technol. 2, 9 (2016)

9. J. Whan Bae, C.E. Singer, K.D. Huf, Synergistic spent nuclear fuel dynamics within the European Union, Progr. Nucl. Energy 114, 1-12 (2019)

10. P.L. Joskow, J.E. Parsons, The future of nuclear power after Fukushima, Econ. Energy Environ. Policy 1, 2 (2012)

11. R. Mendelevitch, T.T. Dang, Nuclear Power and the Uranium Market: Are Reserves and Resources Sufficient? No. 98 (2016) DIW Roundup: Politik im Fokus

12. S. Tillement, F. Garcias, ASTRID, back to the future: bridging scales in the development of nuclear infrastructures, Nucl. Technol. 207-9, 1291-1311 (2021)

13. Ministère de la transition écologique et solidaire (France), Programmation Pluriannuelle de l'Energie: 2019-2023, 20242028 (2020)

14. N. Thiollière, J.B. Clavel, F. Courtin, X. Doligez, M. Ernoult, Z. Issoufou, G. Krivtchik, B. Leniau, B. Mouginot, A. Bidaud, S. David, V. Lebrin, C. Perigois, Y. Richet, A. Somaini, A methodology for performing sensitivity analysis in dynamic fuel cycle simulation studies applied to a PWR fleet simulated with the CLASS tool, EPJ Nuclear Sci. Technol. 4, 13 (2018)

15. A.V. Skarbeli, F. Álvarez-Velarde, Uncertainty quantification on advanced fuel cycle scenario simulations applying local and global methods, Ann. Nucl. Energy 124, 349-356 (2019)
16. B. Feng, S. Richards, J. Bae, E. Davidson, A. Worrall, R. Hays, Sensitivity and Uncertainty Quantification of Transition Scenario Simulations, Argonne National Lab. (ANL) ANL/NSE-20/38 (2020)

17. W. Zhou, G. Krivtchik, P. Blaise, Resilience of nuclear fuel cycle scenarios: Definition, method and application to a fleet with uncertain power decrease, Int. J. Energy Res. 45-8, 12173-12194 (2020)

18. J. Liang, M. Ernoult, X. Doligez, S. David, S. Bouneau, N. Thiollière, G. Krivtchik, F. Courtin, W. Zhou, S. Tillement, Assessment of strategy robustness under disruption of objective in dynamic fuel cycle studies, Ann. Nucl. Energy 154, 108131 (2021)

19. G. Youinou, A. Vasile, Plutonium multirecycling in standard PWRs loaded with evoutionary fuels, Nucl. Sci. Eng. 151, 25-45 (2005)

20. G. Martin, M. Guyot, F. Laugier, G. Senentz, G. Krivtchik, B. Carlier, D. Lecarpentier, F. Descamps, C. Chabert, R. Eschbach, French Scenarios Toward Fast Plutonium Multirecycling in PWR, in ICAPP 2018 (Charlotte, United States, 2018), 103-112

21. F. Courtin, N. Thiollière, X. Doligez, M. Ernoult, B. Leniau, J. Liang, B. Mouginot, A.-A. Zakari-Issoufou, Assessment of plutonium inventory management in the French nuclear fleet with the fuel cycle simulator CLASS, Nucl. Eng. Des. 377, 111042 (2021)

22. J.A. Nelder, R. Mead, A simplex method for function minimization, Comput. J. 7, 308-313 (1965)

23. S. Singer, S. Singer, Efficient implementation of the NelderMead search algorithm, Appl. Numer. Anal. Comput. Math. 1, 524-534 (2004)

24. B. Leniau, B. Mouginot, N. Thiollière, X. Doligez, A. Bidaud, F. Courtin, M. Ernoult, S. David, A neural network approach for burn-up calculation and its application to the dynamic fuel cycle code CLASS, Ann. Nucl. Energy 81, 125-133. (2015).

25. A.-A. Zakari-Issoufou, M. Ernoult, X. Doligez, Consequences on using macro power reactors in nuclear scenarios, in 3rd Technical Workshop On Fuel Cycle Simulation (2018)

26. F. Courtin, Etude de l'incinération du plutonium en REP MOX sur support d'uranium enrichi avec le code de simulation dynamique du cycle CLASS, $\mathrm{PhD}$ thesis, Ecole nationale supérieure Mines-Télécom Atlantique, 2017, French

27. M. Ernoult, X. Doligez, N. Thiollière, A.A. Zakari-Issoufou, A. Bidaud, S. Bouneau, J.B. Clavel, F. Courtin, S. David, A. Somaini, Global and flexible models for sodium-cooled fast reactors in fuel cycle simulations, in PHYSOR 2018: Reactor Physics paving the way towards more efficient systems, Cancun, Mexico (2018)

Cite this article as: Jiali Liang, Marc Ernoult, Xavier Doligez, Sylvain David, Nicolas Thiollière, Impact of disruption between options of plutonium multi-recycling in PWRs and in SFRs, EPJ Nuclear Sci. Technol. 7, 20 (2021) 